\title{
Characterization of ARVC substrate on MRI and electrophysiological mapping
}

\author{
Stephanie Clement-Guinaudeau*, Marjorie Salel, Olivier Corneloup, Gael Dournes, Michel Montaudon, \\ François Laurent, Hubert Cochet \\ From 19th Annual SCMR Scientific Sessions \\ Los Angeles, CA, USA. 27-30 January 2016
}

\section{Background}

In patients with ARVC, breath-hold late gadoliniumenhanced imaging (LGE) may lack spatial resolution to assess the dysplasic substrate on the thin RV wall. We used electrophysiological contact mapping to evaluate the accuracy of breath-hold LGE (BH-LGE), free-breathing LGE (FB-LGE) and cine imaging in describing the extent of dysplasic substrate in ARVC.

\section{Methods}

We studied consecutive patients with definite ARVC diagnosis according to Task Force criteria (TFC), undergoing electrophysiological study for ventricular tachycardia. CMR imaging was performed on a $1.5 \mathrm{~T}$ system (Avanto, Siemens, Erlangen, Germany). SSFP cine imaging was performed in 2 stacks of contiguous $6 \mathrm{~mm}$-thick slices encompassing the whole ventricles in short axis and 4-chamber orientations. BH-LGE imaging was performed $10 \mathrm{~min}$ after the injection of $0.2 \mathrm{mmol} / \mathrm{Kg}$ gadoterate meglumine using a 3D turbo FLASH sequence in 3 stacks of contiguous $6 \mathrm{~mm}$-thick slices encompassing the whole ventricles in short axis, 2-chamber and 4-chamber orientations (pixel size $1.6 \times 1.6 \times 6 \mathrm{~mm}$ ). FB-LGE acquisition was initiated 15 min after contrast using an inversion recovery-prepared and respiratory navigated 3D Turbo FLASH sequence with fat saturation, in order to acquire a whole heart volume at higher spatial resolution (pixel size $1.25 \times 1.25 \times 2.5 \mathrm{~mm}$ ). Wall motion abnormalities (WMA) and LGE were assessed by 2 observers analyzing the images in consensus. This substrate was distributed over a biventricular 16-segment model: the RV and LV free walls comprised 7 segments each ( 3 basal, 3 midventricular and 1 apical), and the septum comprised 2 segments (basal and midventricular). All patients underwent electrophysiological contact mapping during sinus rhythm on RV endocardium and RV and LV epicardium. Low bipolar voltage and local abnormal ventricular activity (LAVA) were distributed over the same segmentation.

\section{Results}

23 patients were included (age $45 \pm 14$ years, 5 women). MRI was positive for a major TFC in $14 / 23(61 \%)$, for a minor TFC in $3 / 23(13 \%)$, and it was negative in $6 / 23$ patients (26\%). RVEF and RVEDV were $38 \pm 9 \%$ and 111 $\pm 24 \mathrm{~mL} / \mathrm{m}^{2}$, respectively. LVEF and LVEDV were $57 \pm$ $11 \%$ and $82 \pm 18 \mathrm{~mL} / \mathrm{m}^{2}$, respectively. On the RV, low voltage and/or LAVA were found at contact mapping in all patients, WMA in $22 / 23(96 \%)$, FB-LGE in $20 / 23(87 \%)$, and BH-LGE in 16/23(70\%). On regional analysis, WMA and FB-LGE showed higher agreement with contact mapping than BH-LGE ( $\mathrm{k}=0.72$ and $\mathrm{k}=0.70$ vs. $\mathrm{k}=0.55$, respectively). On the LV, low voltage and/or LAVA were found at contact mapping in $13 / 23(57 \%)$ patients, WMA in $7 / 23$ (30\%), FB-LGE in 13/23(57\%) and BH-LGE in 13/23(57\%). On regional analysis, FB- and BH-LGE showed higher agreement with contact mapping than WMA $(\mathrm{k}=0.77$ and 0.77 vs. $\mathrm{k}=0.32$ ).

\section{Conclusions}

In patients with proven ARVC, cine imaging is more efficient in detecting dysplasic substrate than the conventional BH-LGE method on the RV, while FB-LGE imaging performs better. Conversely, LGE imaging is more efficient in detecting dysplasic substrate than cine imaging on the LV. 

and take full advantage of:

- Convenient online submission

- Thorough peer review

- No space constraints or color figure charges

- Immediate publication on acceptance

- Inclusion in PubMed, CAS, Scopus and Google Scholar

- Research which is freely available for redistribution 